\title{
Quercetin Upregulates Uncoupling Protein 1 in White/Brown Adipose Tissues through Sympathetic Stimulation
}

\author{
Hyunjung Choi, Chu-Sook Kim, Rina Yu* \\ Department of Food Science and Nutrition, University of Ulsan, Ulsan, Korea
}

Background: Uncoupling protein 1 (UCP1) plays an important role in increasing energy expenditure; thus, it is being considered as a new target for preventing obesity and metabolic complications. In this study, we investigated the effect of quercetin, a naturally occurring flavonoid, on UCP1 expression in white/brown adipose tissues (WAT/BAT).

Methods: Mice were fed a high-fat diet (HFD) supplemented with or without dietary quercetin for 9 weeks, and 3T3-L1 adipocytes were treated with quercetin. Expression of UCP1 and other thermogenic genes/proteins was measured by real-time polymerase chain reaction and/or Western blotting.

Results: Dietary quercetin supplementation increased the level of UCP1 in both WAT and/or BAT of HFD-fed obese mice, which was accompanied by upregulated mRNA levels of thermogenesis-related genes. Quercetin supplementation enhanced the plasma norepinephrine level and tended to upregulate $\beta$-adrenergic receptor mRNA level in the WAT of HFD-fed obese mice, accompanied by AMP-activated protein kinase (AMPK) activation. Moreover, quercetin enhanced UCP1 expression in 3T3-L1 adipocytes, and this was blunted by treatment with a peroxisome proliferator-activated receptor gamma (PPAR $\gamma$ ) antagonist.

Conclusion: These findings suggest that quercetin upregulates UCP1, implying increased WAT browning and BAT activity, via activation of the AMPK/PPAR $\gamma$ pathway through sympathetic stimulation. Quercetin may be useful for preventing obesity and metabolic complications.

Key words: Obesity, Adipose tissue, Uncoupling protein 1, Quercetin

\author{
Received February 5, 2018 \\ Reviewed March 5, 2018 \\ Accepted April 9, 2018 \\ ${ }^{*}$ Corresponding author \\ Rina Yu \\ https://orcid.org/0000-0002-1965-3891 \\ Department of Food Science and \\ Nutrition, University of Ulsan, 93 \\ Daehak-ro, Nam-gu, Ulsan 44610, Korea \\ Tel: +82-52-259-2372 \\ Fax: +82-52-259-1698 \\ E-mail: rinayu@ulsan.ac.kr
}

\section{INTRODUCTION}

Obesity increases risks of metabolic complications such as insulin resistance and type 2 diabetes. Adipose tissues play an important role in energy storage, energy dissipation, and endocrine function, and an excessive accumulation of fat in adipose tissue, which results from an imbalance between energy intake and energy expenditure, causes chronic low-grade inflammation and hence is thought to be a major contributor to the pathogenesis of obesity. ${ }^{1,2}$
Adipose tissues are classified into two different types: white adipose tissue (WAT) and brown adipose tissue (BAT). The former is the primary site for storage of excess fat, and the latter is responsible for non-shivering thermogenesis, in which energy is dissipated as heat. There is growing evidence that white adipocytes can acquire a brown-like adipocyte phenotype, known as browning of WAT, such as that of beige/brite adipocytes in WAT depots, in response to different stimuli including beta-adrenergic stimulation and chronic exposure to cold. ${ }^{3}$ Hence, it is conceivable that control-

Copyright (C) 2018 Korean Society for the Study of Obesity

(a) This is an Open Access article distributed under the terms of the Creative Commons Attribution Non-Commercial License (http://creativecommons.org/licenses/by-nc/4.0/) which permits unrestricted non-commercial use, distribution, and reproduction in any medium, provided the original work is properly cited. 
ling BAT activity and browning of WAT may protect against obesity and its related complications. Uncoupling protein 1 (UCP1), a thermogenic factor, is highly expressed in BAT and is also expressed during beige adipocyte development (browning) in WAT. ${ }^{3}$ UCP1 is located in the mitochondrial inner membrane; it uncouples oxidative phosphorylation from adenosin triphosphate synthesis, thereby dissipating energy by triglyceride hydrolysis, which leads to increased energy expenditure. ${ }^{4}$ The induction of UCP1 accompanied by thermogenesis has been recognized as a new strategy for preventing obesity and metabolic complications. ${ }^{5}$ With respect to this, food-derived thermogenic activators capable of inducing UCP1, which facilitates WAT browning and/or increases BAT activity, may protect against obesity and metabolic complications.

Quercetin is a polyphenolic flavonoid that is present in many fruits and vegetables, including onions, broccoli, tomatoes, and apples. We and others have shown that it elicits protective effects against obesity-induced peripheral/central inflammation and signs of metabolic dysregulation such as insulin resistance, hyperlipidemia, muscle atrophy, and fatty liver. ${ }^{6-9}$ Some plant-derived polyphenolic compounds such as curcumin and resveratrol, which have anti-obesity properties, increase UCP1 expression in BAT, WAT, and skeletal muscle of mice fed a high-fat diet (HFD) ${ }^{10,11}$, indicating that their anti-obesity properties are associated with their thermogenic action. Quercetin has been shown to modulate mitochondrial function ${ }^{12}$, which is important for the thermogenic response. However, quercetin's potential to act as a thermogenic factor by inducing browning of WAT and/or activation of BAT, as well as the underlying mechanisms of the action, have not been thoroughly investigated.

In this study, we demonstrate that quercetin induces UCP1 expression, implicating WAT browning and BAT activation. Quercetin-induced upregulation of UCP1 was associated with activation of the AMP-activated protein kinase (AMPK)/peroxisome proliferator-activated receptor gamma (PPAR $\gamma)$ pathway through sympathetic stimulation.

\section{METHODS}

\section{Chemicals and antibodies}

Quercetin was purchased from Sigma-Aldrich (St. Louis, MO,
USA). Antibodies used for Western blotting were anti-UCP1 (Abcam, Cambridge, UK), anti-PPAR $\gamma$ coactivator 1-alpha (PGC1 $\alpha$, Abcam), anti-PPAR $\gamma$ (Santa Cruz Biotechnology, Santa Cruz, CA, USA), anti-cyclic AMP (cAMP)-dependent protein kinase (PKA, Abcam), anti-phospho-AMPKa (Cell Signaling Technology, Danvers, MA, USA), and anti-AMPKa (Cell Signaling Technology). Anti- $\alpha$-tubulin (Abcam) was used as a control for protein loading.

\section{Animal experiments}

Male C57BL/6 mice were purchased from Orient Bio (Busan, Korea) at 8 weeks old. Experimental animals were housed in a pathogen-free barrier facility that was maintained at $22^{\circ} \mathrm{C} \pm 2{ }^{\circ} \mathrm{C}$ at $55 \%-60 \%$ humidity with a 12-hour light/12-hour dark cycle and free access to autoclaved water. After adapting for 1 week, mice were randomly divided into two groups: one group was fed an HFD (60\% of calories from fat; Research Diets, New Brunswick, NJ, USA; $\mathrm{n}=6$ ) and the other, an HFD supplemented with $0.05 \%$ $(\mathrm{w} / \mathrm{w})$ quercetin ( $\mathrm{HFD}+0.05 \%$ Que, $\geq 95 \%$ purity; $\mathrm{n}=6$ ) for 9 weeks. Body weights and food intakes were measured weekly. At the end of 9 weeks of treatment, mice were sacrificed by carbon dioxide anesthesia. Inguinal WAT and BAT were rapidly isolated and weighed. One side of adipose tissue from each mouse was stored at $-80^{\circ} \mathrm{C}$ until further analyses. A middle portion of the other side was fixed in $10 \%$ formaldehyde for sectioning and staining. All animal experiments were approved by the University of Ulsan Animal Care and Use Committee (No. LNY-15-010).

\section{Histology and immunohistochemistry}

Tissues fixed in $10 \%$ formaldehyde were sectioned ( $5 \mu \mathrm{m}$ thick) after being paraffin embedded. Routine hematoxylin-eosin staining of adipose tissue sections was performed. Immunohistochemistry for the UCP1 was performed on deparaffinized sections. For antigen retrieval, slides were submerged in $0.01 \mathrm{M}$ sodium citrate $(\mathrm{pH}$ 6.0) and heated to $100^{\circ} \mathrm{C}$ for 20 minutes. To remove intrinsic peroxidase, slides were incubated with $3 \% \mathrm{H}_{2} \mathrm{O}_{2}$ for 5 minutes, and to prevent nonspecific reactions, slides were incubated with $1.5 \%$ normal blocking serum for 20 minutes. This step was followed by incubation with rabbit polyclonal anti-UCP1 primary antibody (Abcam) diluted 1:200 in 1.5\% normal blocking serum (Vector, Burlingame, CA, USA) overnight at room temperature. Then, second- 
ary antibody incubation and detection were performed for $30 \mathrm{~min}$ utes with biotinylated goat anti-rabbit antibody (1:200, Vector). At the end of each step, slides were washed with phosphate-buffered saline ( $\mathrm{pH} 7.4)$ for 5 minutes. Signal was detected with a Vector kit (Vector). UCP1-positive areas in random fields in each section were determined by examination of stained areas under a microscope (Carl Zeiss, Gottingen, Germany).

\section{Cell culture and differentiation}

Dulbecco's modified Eagle's medium (Gibco, Grand Island, NY, USA) containing 10\% fetal bovine serum (Biological Industries USA, Cromwell, CT, USA) and $100 \mu \mathrm{g} / \mathrm{mL}$ penicillin-streptomycin (Gibco) was used to culture 3T3-L1 preadipocytes (ATCC, Manassas, VA, USA) at $37^{\circ} \mathrm{C}$ in a $5 \% \mathrm{CO}_{2}$ incubator. Confluent 3T3-L1 preadipocytes $\left(5 \times 10^{4}\right.$ cell/well) in 24-well plates (day 0$)$ were maintained in differentiation induction medium containing $0.5 \mathrm{mM}$ 1-methyl-3-isobutylxanthine (Sigma-Aldrich), $0.25 \mu \mathrm{M}$ dexamethasone (Sigma-Aldrich), $10 \mu \mathrm{M}$ quercetin, and $10 \mu \mathrm{g} / \mathrm{mL}$ insulin (Sigma-Aldrich) for 40-42 hours. Next, cells were maintained in differentiation maturation medium containing $0.5 \mathrm{mM}$ 1-methyl-3-isobutylxanthine, $5 \mu \mathrm{g} / \mathrm{mL}$ insulin, and $10 \mu \mathrm{M}$ quercetin (Jena Bioscience, Jena, Germany) for 6 days, and the culture medium was replaced with fresh medium every 2 days. 3T3-L1 adipocytes treated with $10 \mu \mathrm{M}$ quercetin showed no significant difference in viability compared with control cells. To examine the effects of inhibition of PPAR $\gamma$, cells were pretreated with $20 \mu \mathrm{M}$ GW9662 (PPAR $\gamma$ inhibitor, Sigma-Aldrich) for 1 hour.

\section{Western blot analysis}

Equal amounts of cell samples were dissolved in lysis buffer (150 $\mathrm{mM} \mathrm{NaCl}, 50$ mM Tris-HCl, 1 mM EDTA, 50 mM NaF, 10 mM $\mathrm{Na}_{4} \mathrm{P}_{2} \mathrm{O}_{7}, 1 \%$ IGEPAL, $2 \mathrm{mM} \mathrm{Na}_{3} \mathrm{VO}_{4}, 0.25 \%$ protease inhibitor cocktail, and $1 \%$ phosphatase inhibitor cocktail), separated on sodium dodecyl sulfate-polyacrylamide gel electrophoresis, and transferred onto nitrocellulose membranes. Membranes were blocked and incubated with different antibodies, followed by incubation with secondary antibodies. Membrane proteins were visualized using an enhanced chemiluminescent substrate kit (PerkinElmer, Waltham, MA, USA). The intensity of the protein bands was quantified by densitometry using ImageJ software (National
Table 1. Sequences of primers used for quantitative real-time polymerase chain reaction

\begin{tabular}{|c|c|c|}
\hline Gene & Forward primer & Reverse primer \\
\hline Cidea & CTGTCTCAATGTCAAAGCCACGA & TGTGCAGCATAGGACATAAACCTCA \\
\hline Nif-1 & GACCTTGCCACAGGCAGGTAA & CGCCTGCTCCATGAACACTC \\
\hline$P G C 1 \alpha$ & CCGTAAATCTGCGGGATGATG & CAGTTCGTTCGACCTGCGTAA \\
\hline Prdm16 & CCTAGCCCTGAGCGATACTGTGA & ACAGACAATGGCTGGAATGGTG \\
\hline Tfam & TCAGGAGCAGCAGGCACTACA & CTGAGCTCCGAGTCCTTGAACAC \\
\hline Tmem26 & GGCCGTGAAGCCATAAAGCTA & AAAGGCTCCTGTTGAACCAAGAC \\
\hline UCP1 & АСTGCСАСАССТССАGTСАТT & CTाGCCTCACTCAGGATGGG \\
\hline$\beta 3 A R$ & AAGGGCCGTGAAGATCCAG & TGTTGCATCCATAGCCGTTG \\
\hline Rn18s & TTCCGATAACGAACGAGACTCT & TGGCTGAACGCCACTTGTC \\
\hline
\end{tabular}

Institutes of Health, Bethesda, MA, USA).

\section{Quantitative real-time polymerase chain reaction}

Total RNA was extracted with the TRIzol method (Invitrogen, Carlsbad, CA, USA) following the manufacturer's instructions, and then reverse-transcribed into cDNA using M-MLV reverse transcriptase (Promega, Madison, WI, USA). For target gene expression analysis, cDNA was amplified using a SYBR Premix Ex Taq kit (Takara Bio, Foster City, CA, USA) and a Thermal Cycler Dice (Takara Bio, Kusatsu, Japan). Results were analyzed with Real Time System TP800 software (Takara Bio), and all values for genes were normalized to values for the housekeeping gene, Rn18s. Primers used in the analysis are listed in Table 1.

\section{Statistical analysis}

All data are given as mean \pm standard error of the mean. The significance of differences between the two groups was assessed by Student $t$-test or one-way analysis of variance with Prism 5 (Graphpad, San Diego, CA, USA). Differences were considered to be significant at $P<0.05$.

\section{RESULTS}

\section{Quercetin upregulates UCP1 in WAT and 3T3-L1 adipocytes}

Using HFD-fed obese mice, we first examined whether dietary quercetin supplementation induced expression of UCP1 and browning of WAT in vivo. Quercetin did not affect food intake, though mice that received quercetin had a tendency to gain less 


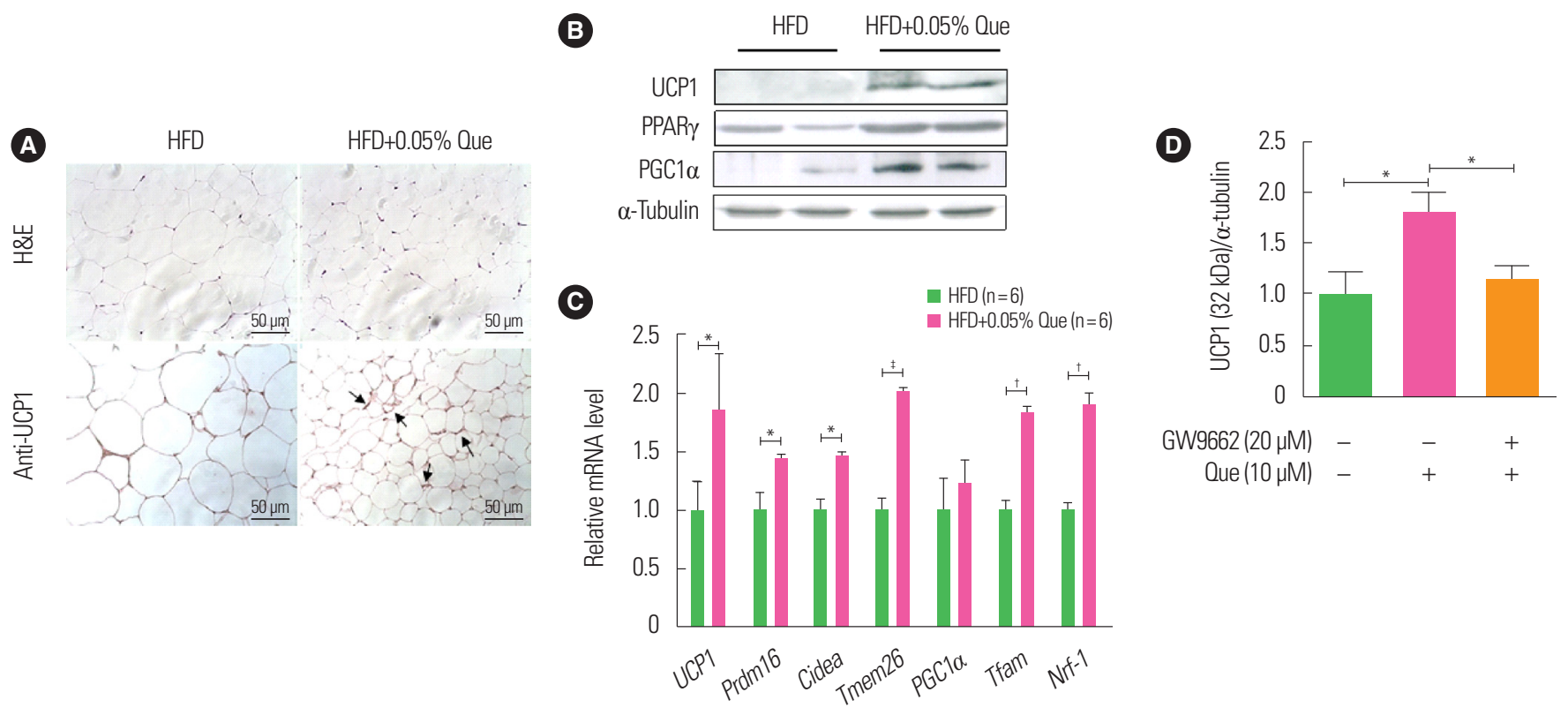

Figure 1. Dietary quercetin enhances expression of uncoupling protein 1 (UCP1) and thermogenic genes in white adipose tissue (WAT). C57BL/6 mice were fed a high-fat $\operatorname{diet}(\mathrm{HFD}$ ) without or with $0.05 \%$ quercetin (HFD or HFD+Que) for 9 weeks. (A) Sections obtained from WAT (inguinal adipose tissue) were stained with H\&E and an antiUCP1 antibody. Original magnification, $\times 200$. The arrows indicate increased UCP1 staining. (B) A representative Western blot showing protein expression levels of UCP1, peroxisome proliferator-activated receptor gamma (PPAR $\gamma$ ), and PPAR $\gamma$ coactivator 1-alpha (PGC1 $\alpha$ ) in inguinal adipose tissue. (C) Expression of thermogenic markers and mitochondrial biogenesis markers were measured by real-time polymerase chain reaction. Results are mean \pm standard error of the mean (SEM; $n=6$ mice per group). ${ }^{*} P<0.05 ;{ }^{\dagger} P<0.01 ;{ }^{\ddagger} P<0.001$ compared with control obese mice. (D) 3T3-L1 adipocytes were treated with or without quercetin and/or GW9662 (PPAR $\gamma$ antagonist). Results are mean \pm SEM of three independent experiments. ${ }^{*} P<0.05$ compared with control.

body weight than HFD-fed obese controls, as previously reported. ${ }^{6,7}$ Quercetin supplementation reduced WAT weight (data not shown) and the size of white adipocytes compared to HFD-fed obese controls (Fig. 1A). Immunohistochemical analysis of UCP1 in WAT revealed that there were more clusters of UCP1-expressing smaller adipocytes in WAT from the quercetin-supplemented HFD-fed obese group than WAT from the HFD-fed obese controls (Fig. 1A). In addition, Western blotting showed that expression of UCP1 was significantly upregulated by quercetin supplementation in HFD-fed obese mice (Fig. 1B). Subsequently, we found that protein levels of browning markers, namely PPAR $\gamma$ and PGC1 $\alpha$, were enhanced in WAT from quercetin-supplemented HFD-fed obese mice (Fig. 1B). In addition, we found that the expression of thermogenesis-related genes, including transcription factor A (Tfam), nuclear respiratory factor-1 (Nrf-1), PR domain containing 16 (Prdm16), cyclic adenosine monophosphate ( $\mathrm{Ci}$ dea), and transmembrane protein 26 (Tmem26), was enhanced in WAT from quercetin-supplemented HFD-fed obese mice (Fig. 1C). Next, to test whether the effect of quercetin on UCP1 induc- tion was associated with its ligand action on PPAR $\gamma$, we treated 3T3-L1 adipocytes with quercetin with or without GW9662, a PPAR $\gamma$ antagonist. As shown in Fig. 1D, quercetin treatment significantly upregulated UCP1 in 3T3-L1 adipocytes, and quercetin action was blunted by treatment with GW9662.

\section{Quercetin increases plasma norepinephrine and $\beta 3 A R$ mRNA levels in WAT}

The UCP1-mediated thermogenic response is regulated primarily by the sympathetic nervous system (SNS) through the binding of norepinephrine to the $\beta 3$-adrenergic receptor ( $\beta 3 A R)$. We examined whether quercetin affects norepinephrine release and the expression of its receptor. As shown in Fig. 2A, levels of plasma norepinephrine in quercetin-supplemented HFD-fed obese mice were significantly higher than in HFD-fed obese controls. Additionally, we observed that quercetin supplementation tended to upregulate levels of $\beta 3 A R$ mRNA in WAT from HFD-fed obese mice as compared with HFD-fed obese controls (Fig. 2B). 


$$
\text { A }
$$
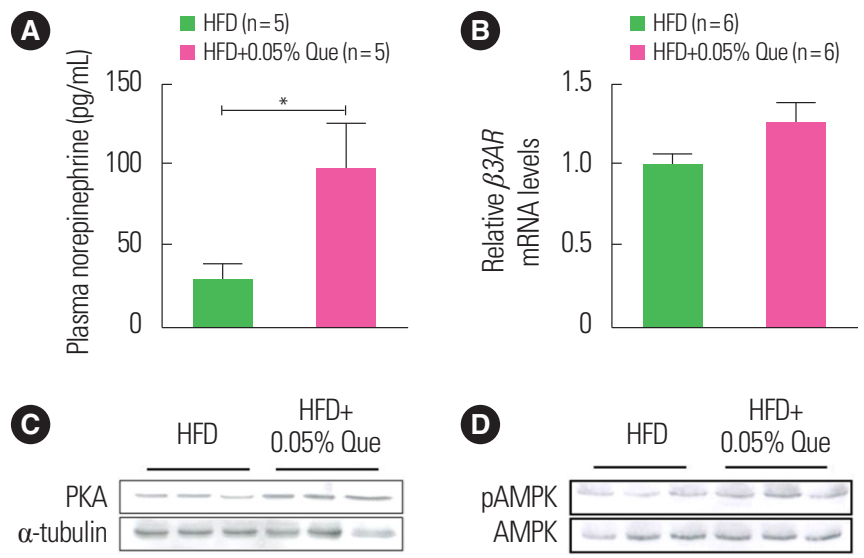

$\operatorname{HFD}(\mathrm{n}=5)$

HFD+0.05\% Que $(n=5)$

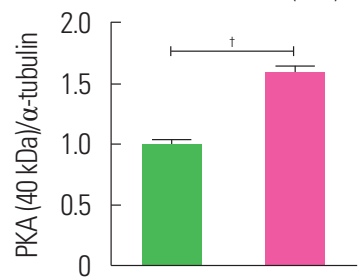

A
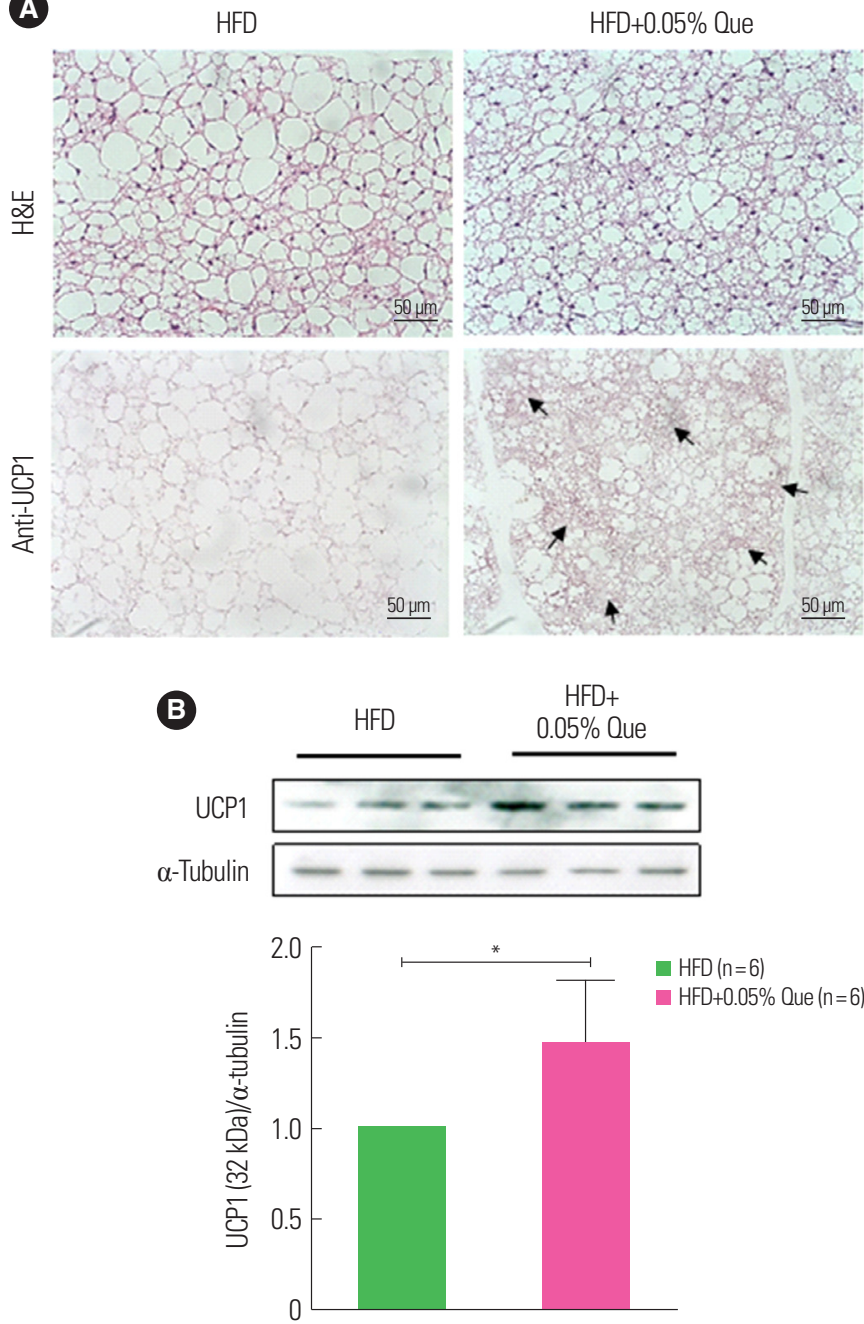

Figure 3. Dietary quercetin increases uncoupling protein 1 (UCP1) expression in brown adipose tissue (BAT). C57BL/6 mice were fed a high-fat diet (HFD) without or with $0.05 \%$ quercetin (HFD or HFD+Que) for 9 weeks. (A) Sections obtained from BAT were stained with H\&E and an anti-UCP1 antibody. Original magnification, $\times 200$. The arrows indicate increased UCP1 staining. (B) A representative Western blot showing protein expression of UCP1 in BAT. Results are mean \pm standard error of the mean ( $\mathrm{n}=6$ mice per group). ${ }^{*} P<0.05$ compared with control.

expenditure. To examine the effect of quercetin supplementation on BAT activity, we measured UCP1 expression in the BAT of HFD-fed obese mice. BAT from quercetin-supplemented HFDfed obese mice contained small brown adipocytes with multiple small lipid droplets and a centralized nucleus surrounded by a clear cytoplasm, compared to HFD-fed controls (Fig. 3A). In addition, there was stronger immunostaining of UCP1-positive areas in quercetin-supplemented HFD-fed mice than control mice (Fig. 3B). Consistent with this, Western blotting showed that expression of the UCP1 protein in BAT was significantly upregulated by quer- 
cetin supplementation (Fig. 3B).

\section{DISCUSSION}

Upregulation of UCP1 mRNA/protein, which is closely associated with increased adaptive thermogenic activity and energy expenditure ${ }^{14}$, is widely used as a marker not only of BAT activity, but also of a brown-like phenotype, such as that of beige/brite adipocytes. ${ }^{15}$ Studies have shown that several flavonoids have thermogenic activity by inducing UCP1 in BAT and WAT. ${ }^{10,11}$ In this study, we found that quercetin supplementation enhanced the expression of a core set of thermogenic genes (i.e., UCP1, Cidea, Tmem26) in the WAT of HFD-fed obese mice. More importantly, quercetin markedly upregulated UCP1 at the protein level in WAT and/or BAT of HFD-fed obese mice as well as in 3T3-L1 adipocytes. Consistent with this, $\operatorname{Prdm16}$, a transcriptional coregulator responsible for browning induction and thermogenic maintenance of the beige adipocyte phenotyp $\mathrm{e}^{16}$ was upregulated in the WAT of quercetin-supplemented HFD-fed obese mice. These findings indicate that quercetin may act as a thermogenic activator and thus may promote the browning of WAT. This action could explain, at least in part, the positive effects of quercetin supplementation on metabolic derangements in obese mice reported previously. ${ }^{6-9}$

It is known that the SNS, which innervates BAT, regulates thermogenic activity by increasing norepinephrine release from BAT SNS terminals. Norepinephrine acts on $\beta 3 A R$ expressed in brown adipocytes, activating mitochondrial UCP1, which is responsible for increased BAT thermogenesis. $\beta$-adrenergic stimulation can enhance not only BAT activation, but also WAT browning ${ }^{17}$, and may therefore protect against obesity and metabolic complications. ${ }^{18,19}$ Indeed, we found that quercetin supplementation increased norepinephrine levels in the plasma of HFD-fed obese mice. In addition, quercetin upregulated $\beta 3 A R$ mRNA levels in the WAT of HFD-fed obese mice. Stimulation of $\beta 3 A R$ in brown and white adipocytes activates adenylyl cyclase to raise levels of intracellular cAMP, whose target is PKA and AMPK, leading to the induction

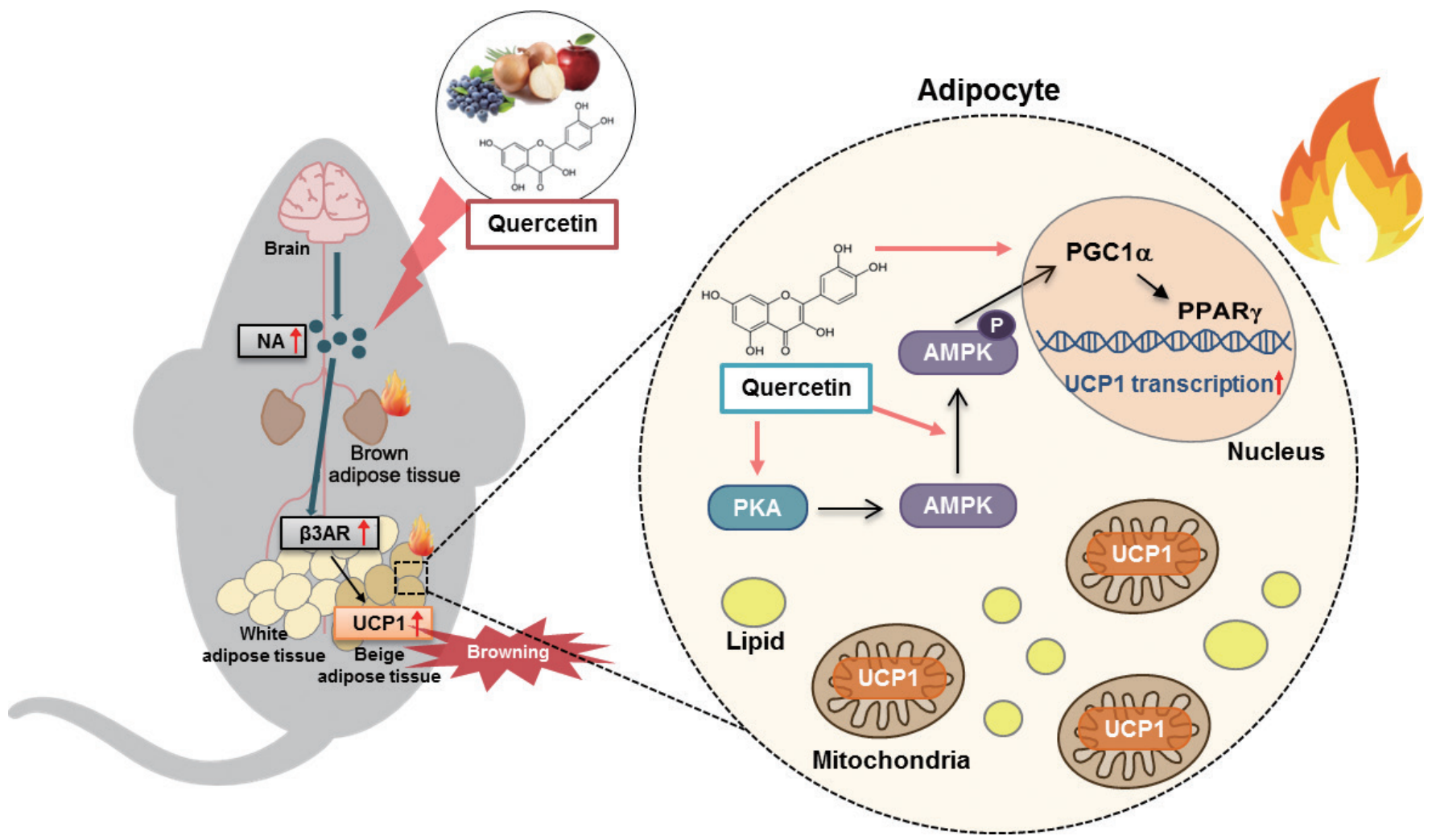

Figure 4. Schematic presentation of quercetin action on uncoupling protein 1 (UCP1) induction. Quercetin enhances UCP1 expression through AMPK/PPAR $\gamma$ activation and sympathetic stimulation, promoting white adipose tissue browning and brown adipose tissue activation. $\beta 3 A R$, $\beta 3$-adrenergic receptor; PPAR $\gamma$, peroxisome proliferator-activated receptor gamma; PGC1 $\alpha$, PPAR $\gamma$ coactivator 1-alpha; AMPK, AMP-activated protein kinase; PKA, cyclic AMP-dependent protein kinase. 
of UCP $1 .{ }^{20,21}$ In this study, we observed that quercetin increased PKA expression in WAT, which is a downstream signaling molecule of $\beta 3 A R$. Subsequently, we confirmed that quercetin increased AMPK phosphorylation in WAT. Taken together, our data suggest that quercetin induces UCP1 expression in adipose tissues (WAT/ BAT) is associated with sympathetic stimulation through $\beta 3 A R$ signaling-induced PKA/AMPK activation.

Expression of UCP1 is directly controlled by $\operatorname{PPAR} \gamma$, a ligandactivated transcription factor of the nuclear receptor superfamily, by interaction with its coactivator PGCla, indicating that PPAR $\gamma$ and PGC1 $\alpha$ are essential for promoting the browning of WAT and the development of $\mathrm{BAT}^{2-22}$ In this study, we observed that quercetin upregulated the expression of PPAR $\gamma$ and PGC1 $\alpha$ proteins in WAT, indicating that quercetin-induced UCP1 induction may be attributed to quercetin's ability to upregulate PPAR $\gamma$ and PGC1 $\alpha$. Of note, activation of AMPK is known to increase the expression of mitochondrial biogenesis signals via PGC1 $\alpha$ expression. ${ }^{25} \mathrm{We}$ found that quercetin enhanced the phosphorylation of AMPK in the WAT of HFD-fed obese mice, and this was accompanied by upregulation of the mitochondria-related genes Tmem 26, Tfam, and Nrf-1. These results indicate that quercetin-induced transcriptional activation of UCP1 can be attributed to enhanced phosphorylation of AMPK. Intriguingly, we observed that a PPAR $\gamma$ antagonist eliminated quercetin-mediated upregulation of UCP1 in 3T3-L1 adipocytes. Taken together, these observations suggest that quercetin-induced browning is partially associated with its ability to activate PPAR $\gamma$ and AMPK.

In summary, quercetin supplementation increased the expression of UCP1 protein in both WAT and BAT in HFD-fed obese mice, and this was accompanied by upregulation of mitochondrial thermogenic genes. Quercetin supplementation enhanced the plasma level of norepinephrine and the mRNA level of its receptor $\beta 3 A R$ in WAT, accompanied by PKA/AMPK activation (Fig. 4). Moreover, quercetin treatment increased UCP1 expression in 3T3$\mathrm{L} 1$ adipocytes, and this action was blunted by a PPAR $\gamma$ antagonist. These findings indicate that quercetin-induced UCP1 is associated with AMPK/PPAR $\gamma$ activation through sympathetic stimulation, promoting WAT browning and BAT activation. Dietary quercetin may therefore be useful to protect against obesity and metabolic dysregulation.

\section{CONFLICTS OF INTEREST}

The authors declare no conflict of interest.

\section{ACKNOWLEDGMENTS}

This work was supported by the SRC program (Center for Food \& Nutritional Genomics Research: No. 2015R1A5A6001906) of the National Research Foundation of Korea (NRF) funded by the Ministry of Education, Science and Technology.

\section{REFERENCES}

1. Hotamisligil GS. Inflammation and metabolic disorders. Nature 2006;444:860-7.

2. Shoelson SE, Lee J, Goldfine AB. Inflammation and insulin resistance. J Clin Invest 2006;116:1793-801.

3. Peschechera A, Eckel J. "Browning" of adipose tissue: regulation and therapeutic perspectives. Arch Physiol Biochem 2013; 119:151-60.

4. Lidell ME, Betz MJ, Enerbäck S. Brown adipose tissue and its therapeutic potential. J Intern Med 2014;276:364-77.

5. Hill JO, Wyatt HR, Peters JC. Energy balance and obesity. Circulation 2012;126:126-32.

6. Kim CS, Yu R. The inhibitory effect of quercetin on adipose tissue inflammation in mice fed on a high-fat diet. Korean J Obes 2014;23:170-8.

7. Le NH, Kim CS, Park T, Park JH, Sung MK, Lee DG, et al. Quercetin protects against obesity-induced skeletal muscle inflammation and atrophy. Mediators Inflamm 2014;2014: 834294.

8. Kim CS, Kwon Y, Choe SY, Hong SM, Yoo H, Goto T, et al. Quercetin reduces obesity-induced hepatosteatosis by enhancing mitochondrial oxidative metabolism via heme oxygenase-1. Nutr Metab (Lond) 2015;12:33.

9. Yang J, Kim CS, Tu TH, Kim MS, Goto T, Kawada T, et al. Quercetin protects obesity-induced hypothalamic inflammation by reducing microglia-mediated inflammatory responses via HO-1 induction. Nutrients 2017;9:650.

10. Wang S, Wang X, Ye Z, Xu C, Zhang M, Ruan B, et al. Cur- 
cumin promotes browning of white adipose tissue in a norepinephrine-dependent way. Biochem Biophys Res Commun 2015;466:247-53.

11. Azhar Y, Parmar A, Miller CN, Samuels JS, Rayalam S. Phytochemicals as novel agents for the induction of browning in white adipose tissue. Nutr Metab (Lond) 2016;13:89.

12. de Oliveira MR, Nabavi SM, Braidy N, Setzer WN, Ahmed T, Nabavi SF. Quercetin and the mitochondria: a mechanistic view. Biotechnol Adv 2016;34:532-49.

13. Pulinilkunnil T, He H, Kong D, Asakura K, Peroni OD, Lee A, et al. Adrenergic regulation of AMP-activated protein kinase in brown adipose tissue in vivo. J Biol Chem 2011;286:8798809.

14. Jeremic N, Chaturvedi P, Tyagi SC. Browning of white fat: novel insight into factors, mechanisms, and therapeutics. J Cell Physiol 2017;232:61-8.

15. Spiegelman BM. Banting lecture 2012: regulation of adipogenesis: toward new therapeutics for metabolic disease. Diabetes 2013;62:1774-82.

16. Mirbolooki MR, Upadhyay SK, Constantinescu CC, Pan ML, Mukherjee J. Adrenergic pathway activation enhances brown adipose tissue metabolism: a [18F]FDG PET/CT study in mice. Nucl Med Biol 2014;41:10-6.

17. Bonet ML, Oliver P, Palou A. Pharmacological and nutritional agents promoting browning of white adipose tissue. Biochim Biophys Acta 2013;1831:969-85.

18. Zhang X, Zhang QX, Wang X, Zhang L, Qu W, Bao B, et al.
Dietary luteolin activates browning and thermogenesis in mice through an AMPK/PGC1 $\alpha$ pathway-mediated mechanism. Int J Obes (Lond) 2016;40:1841-9.

19. Langin D. Recruitment of brown fat and conversion of white into brown adipocytes: strategies to fight the metabolic complications of obesity? Biochim Biophys Acta 2010;1801:372-6.

20. Collins S. $\beta$-Adrenoceptor signaling networks in adipocytes for recruiting stored fat and energy expenditure. Front Endocrinol (Lausanne) 2012;2:102.

21. Collins S, Yehuda-Shnaidman E, Wang H. Positive and negative control of UCP1 gene transcription and the role of $\beta$-adrenergic signaling networks. Int J Obes (Lond) 2010;34 Suppl 1:S2833.

22. Hondares E, Rosell M, Díaz-Delfín J, Olmos Y, Monsalve M, Iglesias $\mathrm{R}$, et al. Peroxisome proliferator-activated receptor $\alpha$ (PPAR $\alpha)$ induces PPAR $\gamma$ coactivator $1 \alpha($ PGC- $1 \alpha)$ gene expression and contributes to thermogenic activation of brown fat: involvement of PRDM16. J Biol Chem 2011;286:43112 22.

23. Nedergaard J, Petrovic N, Lindgren EM, Jacobsson A, Cannon B. PPARgamma in the control of brown adipocyte differentiation. Biochim Biophys Acta 2005; 1740:293-304.

24. Liang H, Ward WF. PGC-1alpha: a key regulator of energy metabolism. Adv Physiol Educ 2006;30:145-51.

25. Fernandez-Marcos PJ, Auwerx J. Regulation of PGC-1 $\alpha$, a nodal regulator of mitochondrial biogenesis. Am J Clin Nutr 2011;93:884S-890S. 